\title{
Intra-Familial Tests of Association between Familial Idiopathic Scoliosis and Linked Regions on 9q31.3-q34.3 and 16p12.3-q22.2
}

\author{
Nancy H. Miller ${ }^{a} \quad$ Cristina M. Justice ${ }^{b}$ Beth Marosy ${ }^{c}$ Kandice Swindle ${ }^{a}$ \\ Yoonhee Kim $^{\text {b }}$ Marie-Hélène Roy-Gagnon ${ }^{d}$ Heejong Sung ${ }^{b}$ Dana Behneman ${ }^{b}$ \\ Kimberly F. Doheny ${ }^{c}$ Elizabeth Pughc Alexander F. Wilson ${ }^{b}$ \\ a Department of Orthopaedic Surgery, University of Colorado Anschutz Medical Campus, Aurora, Colo., and \\ ${ }^{b}$ Genometrics Section, Inherited Disease Research Branch, National Human Genome Research Institute, \\ National Institutes of Health, and ${ }^{\mathrm{C} C e n t e r}$ for Inherited Disease Research, Institute of Genetic Medicine, \\ The Johns Hopkins University School of Medicine, Baltimore, Md., USA; ${ }^{d}$ Department of Social and Preventive \\ Medicine, CHU Sainte-Justine Research Center, University of Montréal, Montréal, Qué., Canada
}

\author{
Key Words \\ Idiopathic scoliosis • Chromosome $9 \cdot$ Chromosome 16 • \\ Genetic heterogeneity $\cdot$ Genetics · Association • \\ Family-based association study $\cdot$ Complex disease
}

\begin{abstract}
Objective: Custom genotyping of markers in families with familial idiopathic scoliosis were used to fine-map candidate regions on chromosomes 9 and 16 in order to identify candidate genes that contribute to this disorder and prioritize them for next-generation sequence analysis. Methods: Candidate regions on $9 q$ and $16 p-16 q$, previously identified as linked to familial idiopathic scoliosis in a study of 202 families, were genotyped with a high-density map of single nucleotide polymorphisms. Tests of linkage for fine-mapping and intra-familial tests of association, including tiled regression, were performed on scoliosis as both a qualitative and quantitative trait. Results and Conclusions: Nominally significant linkage results were found for markers in both candidate regions. Results from intra-familial tests of association and tiled regression corroborated the linkage findings and
\end{abstract}

identified possible candidate genes suitable for follow-up with next-generation sequencing in these same families. Candidate genes that met our prioritization criteria included FAM129B and CERCAM on chromosome 9 and SYT1, GNAO1, and $\mathrm{CDH} 3$ on chromosome 16.

Copyright $\odot 2012$ S. Karger AG, Basel

\section{Introduction}

Idiopathic scoliosis (IS) is the most common spinal abnormality in children and is clinically characterized by a pain-free abnormal spinal curvature in otherwise normal individuals [1]. The variation of presentation, the limited therapeutic options, and the inability to detect individuals at risk for significant progression have led to the establishment of high-cost screening programs. These programs have resulted in higher rates of subspecialty referrals and additional radiographic analyses. Spinal fusion is the recommended treatment option when curve progression is significant. The economic cost attributable to scoliosis within the United States has been estimated to

\section{KARGER}

Fax +4161306 1234

E-Mail karger@karger.ch

www.karger.com (c) 2012 S. Karger AG, Basel

0001-5652/12/0741-0036\$38.00/0

Accessible online at:

www.karger.com/hhe
Nancy Hadley-Miller

Department of Orthopaedic Surgery

University of Colorado Anschutz Medical Campus

12800 E 19th Ave, MS8343, Aurora, CO 80045 (USA)

E-Mail Nancy.Miller@ childrenscolorado.org 
be as high as USD 3 billion per year, and this does not take into account the morbidity in adulthood related to longterm effects of a spinal fusion done at a young age [2].

Genome-wide linkage screens and fine-mapping of candidate loci in families with at least two members with IS, termed familial idiopathic scoliosis (FIS), have identified candidate regions on $6 \mathrm{q}, 10 \mathrm{q}, 18 \mathrm{q}$ [3]; 17p11.2 [4]; 19p13.3, 2q [5]; Xq23-26 [6]; 6p25-22, 6q14-16, 9q32-34, 16q11-12, 17p11-q11 [7]; 8q12 [8]; 9q31.2-q34.2, 17q25.4qtel [9]; and 12pter [10]. Candidate regions on chromosomes 9q32-34 and 19p13 have been independently confirmed $[9,11]$. Recently, a common variant near $L B X 1$ on $10 \mathrm{q} 24.31$ was found to be associated with adolescent IS in unrelated individuals in two Asian populations $[12,13]$.

In this study, a set of families with FIS was genotyped with two different high-density custom oligonucleotide panels of single nucleotide polymorphisms (SNPs) in order to identify candidate genes and prioritize them for next-generation sequence analysis.

\section{Participants and Methods}

\section{Study Population}

Written informed consent was obtained from all study participants, in accordance with the protocol approved by the Johns Hopkins School of Medicine Institutional Review Board. The study population was comprised of Caucasian families with two or more individuals in the family with IS, and all family members participating in the study were ascertained and examined by a single orthopedic surgeon. Characterization of the study population was performed to document the uniformity and/or variation within the sample population. Parameters of gender, curve type, and size within this familial study population were consistent with previous reports in the literature [14]. The number of affected females exceeded the number of affected males ( 270 vs. 110 ), and the mean curve severity of the females was greater than that of the males $(35.0 \pm 1.2$ vs. $26.9 \pm 2.0)$. The primary curve pattern represented was the single right thoracic curvature [14]. The criteria for a diagnosis of IS were a history and physical examination consistent with a sagittal spinal curvature, and standing anteroposterior spinal radiographs exhibiting $\geq 10^{\circ}$ curvature in the coronal plane by the Cobb method, with pedicle rotation and no congenital deformity [15]. The threshold of $10^{\circ}$ is based on the fact that a graph of scoliosis prevalence among the general population is a smooth exponential function where the sharpest change in the slope occurs at $10^{\circ}$ curvature in the coronal plane [15]. While the initial threshold criteria of $10^{\circ}$ for the definition of scoliosis has proven to be clinically relevant, the significance of this threshold is unknown with respect to the underlying genetics; therefore, additional thresholds of $\geq 20^{\circ}, \geq 30^{\circ}$, and $\geq 40^{\circ}$ were considered. Radiographic measurements of the proband within each family were taken at the time of inclusion into the study and varied from age 8 years to age 16 years with curve measurements of $16-88^{\circ}$. Radiographs of family members were obtained either from historical radiographs or standing spinal ra- diographs at the time of their inclusion into the study. A single orthopedic surgeon performed all radiographic measurements. Measurements related to scoliotic spinal curvatures from radiographs have been well studied for intra-observer consistency [16, 17]. Historical evidence or clinical signs of conditions, including blood clots, cardiac defects, osteoporosis, and known hereditary disorders, in any individual excluded the family from the study. In order to avoid misclassification, individuals without radiographic information were classified as unknown. For individuals with two or more curves, the degree of curvature was obtained from the curve with the largest observed Cobb angle. In addition to the degree of lateral curvature, variables measured included the type of curve, age at diagnosis, ethnic background, awareness of condition, presence of pain, and type of treatment.

In this study, the sample population was genotyped and finemapping linkage analysis and intra-familial tests of association were performed in order to identify and prioritize candidate genes for next-generation sequence analysis. The sample consisted of 544 individuals belonging to a group of 95 families determined most likely to be segregating as an autosomal dominant form of FIS; the average family size was 5.7 individuals, with a range of $2-29$, and 358 (65.9\%) of these 544 individuals were female. Genotype and phenotype information was available on 510 (93.75\%) of these individuals, yielding a missing rate of $6.25 \%$. This sample was genotyped, with SNPs located in the previously identified candidate regions on chromosome 9 (between STRP markers D9S930 and D9S1826 spanning $24 \mathrm{Mb}$ : 115.2-138.4 Mb) and on chromosome 16 (between STRPs D16S764 and D16S2624 spanning $54 \mathrm{Mb}$ : 23.0-54.8 Mb) [7].

\section{Genotype Analysis}

Blood samples were previously obtained from all participants. Genomic DNA was extracted with standard purification protocols [18]. The SNP panels were generated with the National Center for Biotechnology Information (NCBI) dbSNP (http://www.ncbi. nlm.nih.gov/projects/SNP) chromosome report. Custom oligo pools for fine-mapping for linkage analysis and tests of intra-familial associations were designed for the candidate regions on chromosomes 9 and 16 . The panel was designed with special attention to intragenic positioning, linkage disequilibrium blocks, and genes that may have a relationship to spinal growth and development.

SNPs were genotyped using the Illumina BeadArray platform $[19,20]$. The SNPs were genotyped at the Center for Inherited Disease Research (CIDR), McKusick/Nathans Institute of Medicine, The Johns Hopkins University School of Medicine. The Illumina BeadStudio software was used to cluster all SNPs using samples in this study. The SNP plots were manually evaluated and clustered as needed. SNPs were not included if they had poorly defined clusters, excessive replicate or Mendelian errors, and/or more than 50\% missing data. Genotypes were identified with a GenCall score, a quantitative measure that ranges from 0 to 1 and reflects the proximity within a cluster plot of the intensities of that genotype to the centroid of the nearest cluster. All SNPs with a GenCall score $<0.25$ were dropped.

A total of 1,324 SNPs were released for chromosomes 9 and 16 (table 1). As reported by CIDR, the missing, Mendelian inconsistency, and duplicate error rates were $1.4,0.06$, and $0.05 \%$, respectively, for the SNPs genotyped. 
Table 1. Characteristics of SNPs on $9 \mathrm{q} 31-34$ and $16 \mathrm{p} 12-16 \mathrm{q} 22$

\begin{tabular}{lll}
\hline & Chromosome 9 & Chromosome 16 \\
\hline SNPs released & 519 & 805 \\
Average spacing, kb & 46 & $66^{\mathrm{b}}$ \\
Location & $9 \mathrm{q} 31.3-34.3$ & $16 \mathrm{p} 12.3-\mathrm{q} 22.2$ \\
SNP boundaries, $\mathrm{bp}^{\mathrm{a}}$ & $114552025-138460445$ & $18074463-71258937$
\end{tabular}

a Map positions obtained from UCSC website, build GRCh37 (www.genome.ucsc.edu). ${ }^{\mathrm{b}}$ Includes the centromere.

\section{Statistical Genetic Analysis}

Allele frequencies were estimated in the founders with FREQ [21]. Familial relationships were verified with RELCHECK [22, 23], which infers the most likely relationship between pairs of relatives by using estimates of identical by descent (IBD) sharing. Genotyping data were checked for Mendelian inconsistencies with PEDCHECK [24], and non-systematic inconsistencies were removed from the data. Physical positions for the SNPs were obtained from the NCBI dbSNP database (Build 131). For each sibling pair, the proportion of alleles shared IBD was estimated using GENIBD [21].

Monomorphic SNPs, SNPs with a missing rate $>10 \%$, and SNPs for which the minor allele frequency (MAF) was $<1 \%$ were removed prior to analysis. Tests of Hardy-Weinberg equilibrium [25] identified 2 SNPs on chromosome 9 and 4 SNPs on chromosome 16 with $p$ values $<0.001$. Two of these SNPs, 1 on chromosome 9 and 1 on chromosome 16, were dropped due to genotyping errors. Because these are highly ascertained data, the other SNPs were flagged and retained for subsequent analysis. This reduced the number of released SNPs to 1,252, with 500 and 752 on chromosomes 9 and 16, respectively.

Scoliosis was analyzed both as a qualitative and quantitative trait; the qualitative measure represents a clinically relevant classification, and the quantitative measure represents the variation of the degree of lateral curvature. For scoliosis treated as a qualitative trait, the presence or absence of scoliosis was determined by a threshold value used to dichotomize the degree of lateral curvature. This threshold value allows for the conversion of a quantitative trait into a qualitative trait dichotomized into affected and unaffected classes, set by a clinically relevant, although arbitrary, threshold. For the qualitative trait, thresholds of $\geq 10^{\circ}, \geq 20^{\circ}$, $\geq 30^{\circ}$, and $\geq 40^{\circ}$ were considered. For scoliosis treated as a quantitative trait, both untransformed and transformed measurements of the degree of lateral curvature were considered in the analysis. This distribution of the degree of lateral curvature is highly skewed and kurtotic with a substantial probability mass at 0 . Although several transformations were considered (e.g., natural log, Box, and Cox) prior to genetic analysis, none sufficiently normalized the distribution with respect to both skewness and kurtosis. The natural log transformation was chosen because it best discriminated between individuals with a curve of 0 versus a curve greater than 0 and represented a mixture of two distributions.

SNPs in high linkage disequilibrium (LD) must have highly correlated results, both for linkage analysis and for intra-familial tests of association. Including SNPs in high LD increases the number of non-independent tests, provides little, if any, additional information, and inflates the type I error rate. SNPs in high LD were identified by calculating the pairwise LD measures $\mathrm{r}^{2}$ and $\mathrm{D}^{\prime}$ with Haploview [26]. Any pair of SNPs with $\mathrm{D}^{\prime}=1.0$ and $\mathrm{r}^{2}>0.4$ were defined as being in LD, and only the most informative SNP from the pair was retained. The final number of SNPs in these analyses was 846, with 352 and 494 SNPs on chromosomes 9 and 16 , respectively.

\section{Linkage Analysis}

Prior to multi-point linkage analysis, the 846 SNP genotypes were merged with 13 STRP genotypes from the previous genomic screen (6 and 7 on chromosomes 9 and 16, respectively) [7]. Model-independent single-point and multi-point linkage analyses were performed using SIBPAL [21]. The traditional HasemanElston analysis for quantitative and qualitative traits was performed on full-sib relationships. For multi-point linkage analysis, the genetic distances (in $\mathrm{cM}$ ) were assumed to be a linear function of the map distance (in $\mathrm{Mb}$ ). Results from SIBPAL linkage analysis are presented as a p-plot, which plots the log of the inverse of the $\mathrm{p}$ value, or minus the $\log (\mathrm{p})$, against the location of each marker, as a linear function of the map distance [27].

\section{Intra-Familial Tests of Association}

Intra-familial tests of association were carried out on 846 SNPs using FBATv2.0.2c for qualitative traits [28-30] and ASSOC for quantitative traits [21]. FBAT uses data from the members in a nuclear family and can perform tests of association using single SNPs or haplotypes. In order to test for association in these areas of known linkage, the robust empirical variance estimator implemented in FBAT was used, assuming an additive as well as biallelic model [31]. Based on the results from the linkage analysis, individuals with a threshold of $\geq 20^{\circ}$ were classified as affected with FIS for the qualitative trait association analysis.

ASSOC is a likelihood-based test of association that compares the likelihood of the data in models with and without a marker (e.g., a single SNP). Unlike other intra-familial tests, ASSOC uses the phenotype and genotype information of the entire family. The untransformed and natural log transformed $\left(\log _{\mathrm{e}}\right)$ degree of lateral curvature were used as quantitative phenotypes, and a genotypic test was performed. The Bonferroni threshold for the intra-familial tests of association was based on the independent factors (846 SNPs $\times$ three tests of association) and was taken to be 0.00002 .

In addition to single marker association tests, tiled regression was used to identify the set of independent SNPs that are responsible for the variation in the degree of lateral curvature in the context of other SNPs [32-35]. In tiled regression, the genome is divided into independent segments based on predefined regions. Recombination hot spots (i.e., well-defined regions of increased recombination) were used to delineate regions in this study. The term tile denotes both the sequence of DNA between two hot-spot regions and a hot-spot region itself. Each sequence variant is assigned to a tile based on its physical position. A tile is selected if the multiple linear regression on all SNPs in the tile shows a significant relationship to trait variation (testing the null hypothesis that all SNP coefficients are 0) or if the simple linear regression on any single SNP in the tile is significant. A stepwise regression is then used to select the important individual independent SNPs identified in each selected tile. Thereafter, the significant SNPs are combined across tiles in higher-order stepwise regressions 
Fig. 1. Model-independent multi-point linkage analysis results for STRPs and SNPs on chromosome 9.

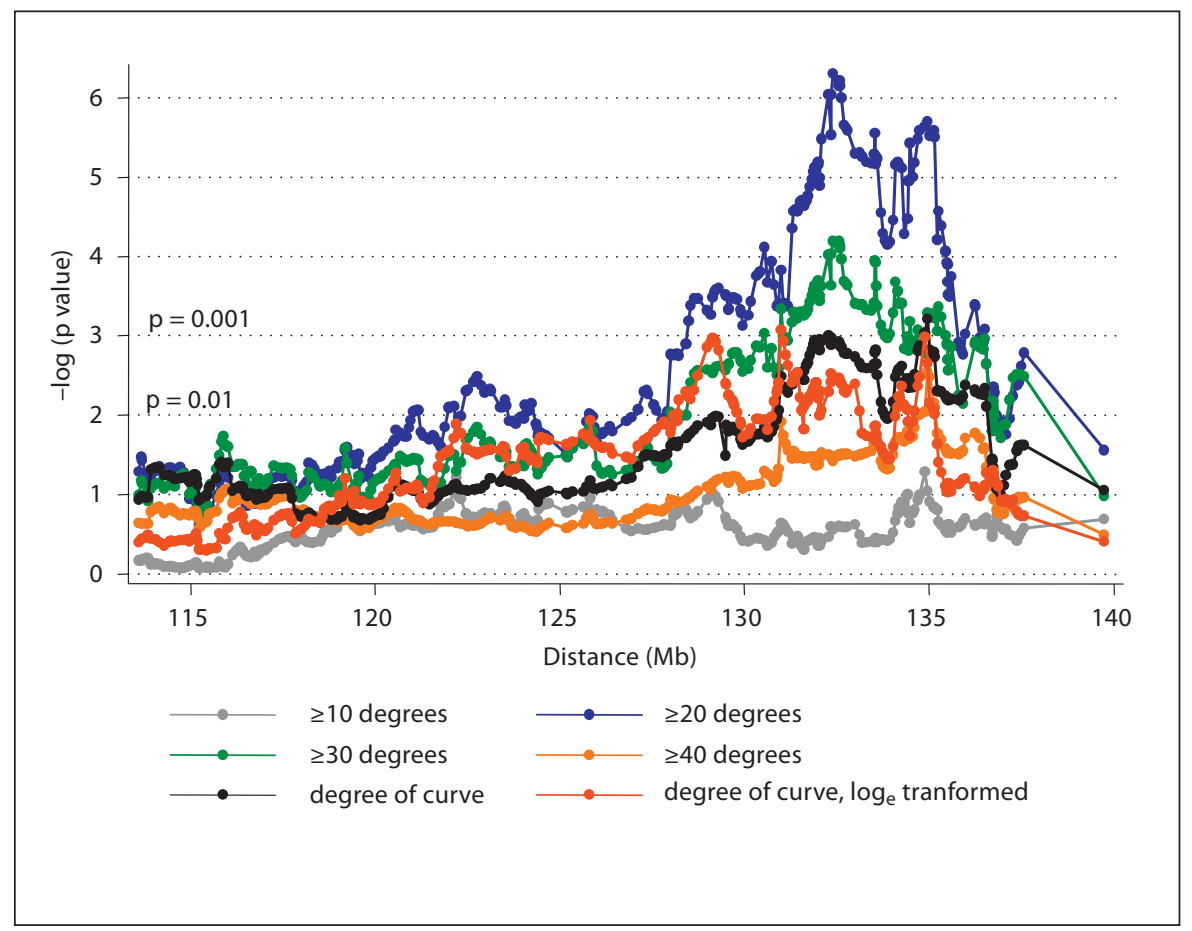

within chromosome and then genome levels. Generalized estimating equations (GEE) [36] were included in the tiled regression framework to allow for familial correlations in families [33]. Sibships were used as the clusters in the GEE covariance matrix estimation, and SNPs with a MAF $<0.05$ were removed prior to analysis. The end result is a multiple linear regression model that includes the set of SNPs that independently contribute to trait variation [35]. Tiled regression was performed with the Tiled Regression Analysis Package (TRAP v 1.0) [34] for the quantitative trait on chromosomes 9 and 16 separately. Critical values of 0.01 and 0.1 were used to select the significant tiles for the simple and multiple linear regressions. A critical value of 0.05 was used to select markers in the stepwise regression. There were 391 markers in 243 tiles on chromosome 9 and 696 markers in 330 tiles on chromosome 16 . All p values reported are nominal values and are not adjusted for multiple tests.

Because different intra-familial tests of association use different kinds of information and test for different types of association as described above, the criterion for prioritizing markers was based on selecting markers that were significant for at least two different methods at a significance level of $\mathrm{p}<0.05$.

\section{Results}

\section{Linkage}

Chromosome 9

Results for the model-independent multi-point linkage analysis on chromosome 9 for both the qualitative and quantitative traits are presented in figure 1 . When the threshold was $\geq 20^{\circ}$, $\mathrm{p}$ values $<0.001$ were found in an $8.0-\mathrm{Mb}$ region (rs3737048 to rs1475718) and p values $<0.0001$ in a $4.2-\mathrm{Mb}$ region (rs968477 to rs753659). At a threshold of $\geq 30^{\circ}$, the linked region was similar to that of the $20^{\circ}$ threshold, but the $\mathrm{p}$ values in this region were not as significant ( $p<0.001$, rs968477 to rs753659) as those in the region based on the $20^{\circ}$ threshold, most likely due to the smaller number of affected individuals at this higher threshold. When the threshold was $\geq 40^{\circ}$, the region with $\mathrm{p}$ values $<0.01$ was less than $0.5 \mathrm{Mb}$ in length (rs944028 to rs568203), which overlaps the region with the most significant $\mathrm{p}$ values $(<0.00001)$ obtained with a threshold of $\geq 20^{\circ}$. No $p$ value $<0.05$ was found when the affection threshold was $\geq 10^{\circ}$. For the quantitative linkage analysis of the transformed and untransformed trait, $p$ values $<0.01$ were found in the same region as identified by qualitative analysis of FIS and spanned about 5.7 and 6.8 $\mathrm{Mb}$ for the untransformed and $\log _{\mathrm{e}}$-transformed traits, respectively (fig. 1).

\section{Chromosome 16}

Evidence for linkage on chromosome 16 spanned the centromere but was strongest on the q-arm region adjacent to the centromere (fig. 2). As with chromosome 9, the significance of the linkage peak increased as the threshold 
Fig. 2. Model-independent multi-point linkage analysis results for STRPs and SNPs on chromosome 16.

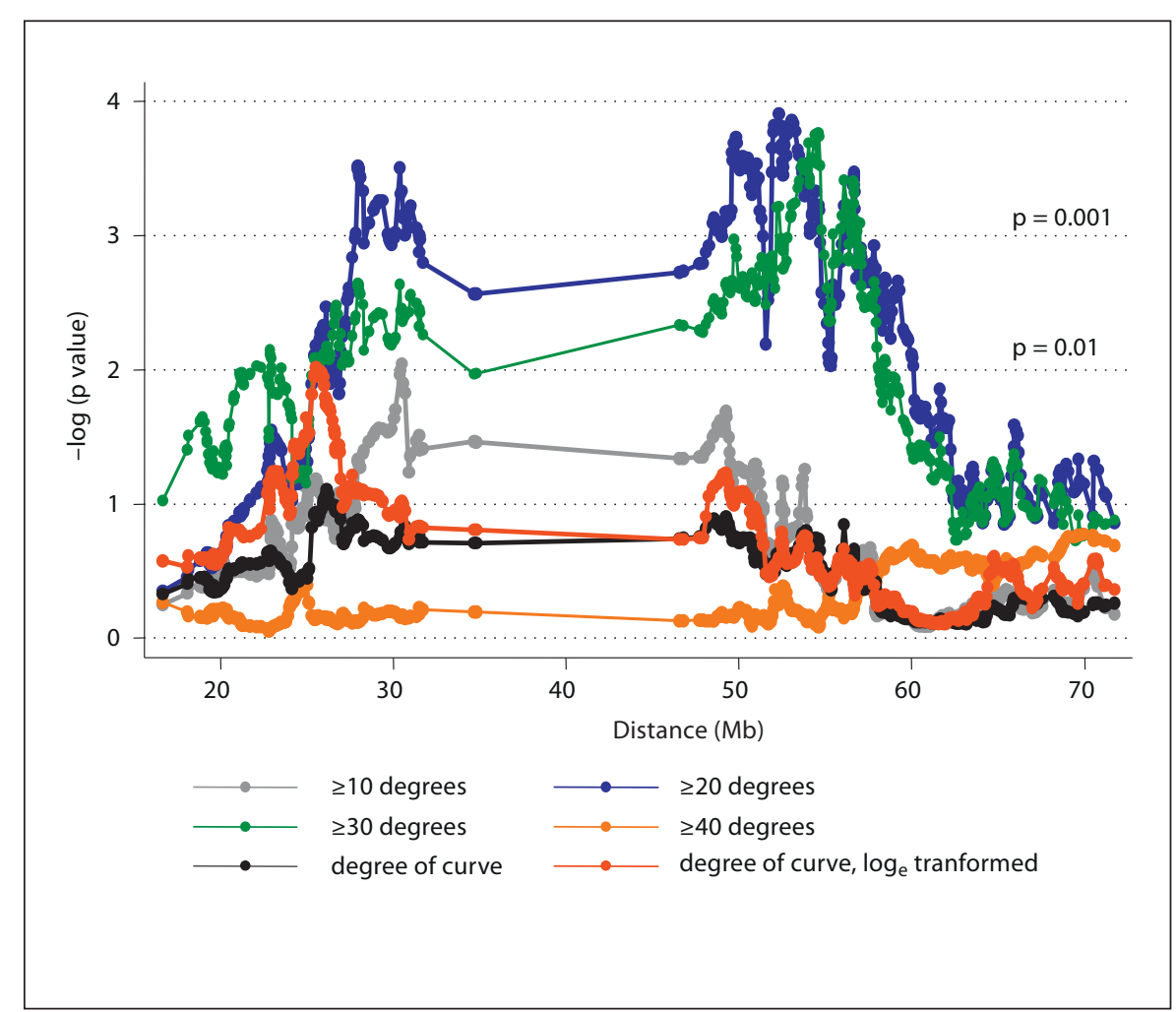

used to dichotomize the qualitative trait increased from $\geq 10^{\circ}$ to $\geq 20^{\circ}$. The most significant $\mathrm{p}$ values were obtained for a threshold of $\geq 20^{\circ}$, with $\mathrm{p}$ values $<0.01 \mathrm{ex}-$ tending over a $34.6-\mathrm{Mb}$ region that included the centromere. $\mathrm{p}$ values $<0.001$ extended over a $3.7-\mathrm{Mb}$ region on $16 \mathrm{p} 11$ (rs713547 to rs3116150) and over 8.2 Mb (rs1566467 to rs8051405) on $16 \mathrm{q} 11$. For the $\geq 30^{\circ}$ threshold, the linkage region on 16p11 was only significant at the 0.01 level, while the peak on $16 \mathrm{q} 11$ remained significant at $\mathrm{p}$ values $<0.001$, and was approximately $4.8 \mathrm{Mb}$ long (rs1420263 to rs 2518054 ). No $p$ values $<0.05$ were found in the analysis of the $\geq 40^{\circ}$ threshold, again most likely due to the small number of individuals taken to be affected with FIS, or for the untransformed quantitative trait. For the $\log _{\mathrm{e}}$-transformed trait, 2 SNPs (rs1022455 and rs508414) on 16p12.1 resulted in $\mathrm{p}$ values $<0.01$, with $\mathrm{p}$ values $<0.05$ extending over a $2.6-\mathrm{Mb}$ region. However, this region was not the same as the region from the qualitative analyses (fig. 2).

\section{Intra-Familial Tests of Association}

Chromosome 9: Qualitative Analysis of FIS with a

$20^{\circ}$ Threshold

For the tests of association for FIS as a qualitative trait with FBAT, 2 SNPs had nominal p values <0.01: rs1306
(132900076 bp, $\mathrm{p}=0.009$ ) located in the $3^{\prime}$-untranslated region of GPR107 (G-protein coupled receptor) and rs1536480 (137412564 bp, p = 0.0093), which is not located within a gene.

Chromosome 9: Quantitative Analysis of the Degree of Lateral Curvature

Only the results for the $\log _{\mathrm{e}}$-transformed trait are presented in the tables, and all significance levels presented are for the nominal significance levels, not adjusted for multiple tests. For ASSOC, 5 SNPs were significant at a level of $p<0.01$ for the $\log _{e}$ transformation (table 2). The most significant association obtained $(\mathrm{p}<0.0014)$ was for $r s 7847869$ located $23 \mathrm{~kb}$ from microRNA MIR3134 (miR3134). For the tiled regression analysis, 6 independent SNPs in 6 tiles were identified. The most significant of these was rs1871692 ( $\mathrm{p}<0.0007)$, which is not located in a known gene but lies in a characterized region indicative of a regulatory function. Significance levels for the SNPs identified with tiled regression are presented in table 2 , and the final multiple regression model was: $\log _{\mathrm{e}}$ $($ degree of lateral curvature +1$)=0.34+0.28 \times$ rs1871692 $+0.19 \times$ rs $944323+0.22 \times$ rs $2249110-0.22 \times$ rs 943392 $-0.28 \times$ rs7259 $-0.19 \times$ rs1537189.
40

Hum Hered 2012;74:36-44 
Table 2. Quantitative tests of association for markers on chromosome 9

\begin{tabular}{lllll}
\hline SNP & bp $^{\mathrm{a}}$ & ASSOC & $\begin{array}{l}\text { Tiled } \\
\text { regression }\end{array}$ & Location \\
\hline rs7847869 & 114736023 & $0.0014^{* *}$ & & \\
rs2676628 & 115369058 & $0.0044^{* *}$ & & intron KIAA1958 \\
rs1871692 & 116501277 & $0.0077^{* *}$ & $0.0007^{* * *}$ & \\
rs944323 & 119674722 & 0.4435 & $0.0349^{*}$ & intron ASTN2 \\
rs2249110 & 130329655 & $0.0053^{* *}$ & $0.0132^{*}$ & intron FAM129B \\
rs943392 & 130725441 & 0.2345 & $0.0279^{*}$ & intron FAM102A \\
rs7259 & 131196704 & $0.0395^{*}$ & $0.0169^{*}$ & CERCAM \\
rs2519760 & 135758421 & $0.0086^{* *}$ & & intron C0orf9 \\
rs1537189 & 137800247 & $0.0141^{*}$ & $0.0256^{*}$ & \\
\hline
\end{tabular}

${ }^{a}$ Map positions from the NCBI dbSNP website, GRCh37.

${ }^{*} \mathrm{p} \leq 0.05 ;{ }^{* *} \mathrm{p} \leq 0.01 ;{ }^{* *} \mathrm{p} \leq 0.001$.

Table 3. Qualitative tests of association for markers on chromosome 16

\begin{tabular}{llll}
\hline SNP & $\mathrm{bp}^{\mathrm{a}}$ & $\mathrm{FBAT} \geq 20^{\circ}$ & Location \\
\hline rs208600 & 22968910 & $0.0064^{* *}$ & \\
rs4889606 & 31011183 & $0.0029^{* *}$ & intron STX1B \\
rs889548 & 31137712 & $0.0073^{* *}$ & intron MYST1 \\
rs7204626 & 51678263 & $0.0058^{* *}$ & \\
rs1111487 & 53562129 & $0.0050^{* *}$ & \\
rs1544806 & 60692954 & $0.0068^{* *}$ & \\
rs2059251 & 60730790 & $0.0004^{* * *}$ & \\
rs1864148 & 64909469 & $0.0006^{* * *}$ & \\
\end{tabular}

${ }^{a}$ Map positions from the NCBI dbSNP website, GRCh37.

${ }^{* *} \mathrm{p} \leq 0.01 ;{ }^{* * *} \mathrm{p} \leq 0.001$.

Four SNPs were significant at the $\mathrm{p}<0.05$ level for both ASSOC and tiled regression: rs1871692 located in a potential regulatory region; rs2249110 located in an intron of the FAM129B gene; rs7259, a synonymous SNP located in the CERCAM gene, and rs1537189 not located within a known gene. No SNP was significant across FIS taken both as qualitative and quantitative trait.

Seven SNPs were significant at a level of $\mathrm{p}<0.01$ for the intra-familial tests of association for the untransformed trait (results not shown). The most significant association obtained was for rs752090 (134550028 bp, p = $0.000002)$. No SNP was significant at the $\mathrm{p}<0.05$ level for both the intra-familial test of association and tiled regression, or across FIS taken both as an untransformed quantitative and qualitative trait.

Association between Chromosomes 9q and $16 \mathrm{p}$ and FIS
Table 4. Summary of quantitative statistical tests for markers on chromosome 16

\begin{tabular}{lllll}
\hline SNP & bp & ASSOC & $\begin{array}{l}\text { Tiled } \\
\text { regression }\end{array}$ & Location \\
\hline rs229018 & 19222713 & $0.0023^{* *}$ & $0.0291^{*}$ & intron SYT17 \\
rs876856 & 19872529 & 0.1123 & $0.0016^{* *}$ & intron GPRC5B \\
rs2214437 & 24275388 & 0.1385 & $0.0329^{*}$ & intron CACNG3 \\
rs723876 & 26886087 & $0.0001^{* * *}$ & $0.0043^{* *}$ & \\
rs1009303 & 49110866 & 0.0531 & $0.0158^{*}$ & \\
rs1861545 & 50499844 & 0.1523 & $0.0058^{* *}$ & \\
rs733017 & 54517482 & 0.0545 & $0.0093^{* *}$ & \\
rs899234 & 56354079 & $0.0310^{*}$ & $0.0005^{* * *}$ & intron GNAO1 \\
rs1864148 & 64909469 & 0.0888 & 0.1450 & \\
rs233551 & 66327313 & 0.1062 & $0.0052^{* *}$ & \\
rs3785133 & 68728611 & $0.0225^{*}$ & $0.0051^{* *}$ & intron CDH3 \\
rs755702 & 64051063 & $0.0071^{* *}$ & & intron LOC283867 \\
rs1423828 & 65536620 & $0.0082^{* *}$ & & intron LOC \\
& & & &
\end{tabular}

${ }^{a}$ Map positions from the NCBI dbSNP website, GRCh37.

${ }^{*} \mathrm{p} \leq 0.05 ;{ }^{* *} \mathrm{p} \leq 0.01 ;{ }^{* *} \mathrm{p} \leq 0.001$.

Chromosome 16: Qualitative Analysis of FIS with a $20^{\circ}$ Threshold

Eight SNPs were significant at a level of $\mathrm{p}<0.01$ for the qualitative trait association tests with FBAT (table 3 ). The most significant result $(\mathrm{p}=0.0004)$ for $\mathrm{rs} 2059251$ was not located in a gene.

Chromosome 16: Quantitative Analysis of the Degree of Lateral Curvature

For the $\log _{\mathrm{e}}$-transformed trait on chromosome 16, 4 SNPs were significant at a level of $\mathrm{p}<0.01$ for ASSOC and 7 SNPs were significant at this same level for tiled regression (table 4). rs723876 was the most significant SNP with $\mathrm{p}<0.001$ for ASSOC but is not located in any known gene. The final multiple regression model for tiled regression was: $\log _{\mathrm{e}}$ (degree of lateral curvature $+1)=-0.43-0.24 \times \mathrm{rs} 229018+0.51 \times \mathrm{rs} 876856+0.19$ $\times$ rs $2214437+0.25 \times \mathrm{rs} 723876-0.25 \times \mathrm{rs} 1009303+$ $-0.31 \times \mathrm{rs} 1861545-0.24 \times \mathrm{rs} 733017-0.46 \times \mathrm{rs} 899234$ $-0.19 \times \mathrm{rs} 1864148+0.44 \times \mathrm{rs} 233551+0.23 \times$ rs3785133.

Four SNPs were significant at the $\mathrm{p}<0.05$ level for both ASSOC and tiled regression: rs229018 located in an intron of SYT17; rs723876 not located in a known gene; rs8999234 located in an intron of the GNAO1 gene, and rs3785133 located in an intron of the $\mathrm{CDH} 3$ gene. No SNP was significant at the $\mathrm{p}<0.05$ level across FIS taken as both qualitative and quantitative trait.

Hum Hered 2012;74:36-44 
For the untransformed trait, 2 SNPs were significant at a level of $\mathrm{p}<0.01$ for ASSOC and 5 SNPs were significant at this same level for tiled regression (results not shown). Three SNPs were significant at the $\mathrm{p}<0.05$ level for both tiled regression and ASSOC: rs723876, rs1009303, and rs3785133 located in an intron of the $\mathrm{CDH} 3$ gene. No SNP was significant at the $\mathrm{p}<0.05$ level across FIS taken as both an untransformed quantitative or qualitative trait.

\section{Discussion}

The genomics era has resulted in major advances in the identification of candidate loci for both sporadic IS and FIS. We previously identified susceptibility loci on multiple chromosomes in a genome-wide linkage scan for FIS in a large study sample [7]. Candidate regions on chromosomes 9q31-34 and 16p-16q have been replicated in distinct study samples and investigations. Ocaka et al. [9] mapped a locus for FIS on chromosome 9q31.2-q34.2 in a British sample, and Sharma et al. [37] reported mild associations to this same region. The area on chromosome 16 has been confirmed by Jose Morcuende [J. Morcuende, pers. commun.] in a sample from the midwestern United States. In the present study, we report results from fine-mapping linkage and intra-familial tests of association analyses of these two regions utilizing custom SNP panels, which corroborated our earlier results and aided in the prioritization of candidate genes for next-generation sequencing in families based on the combined statistical analyses. Because different methods of association use different kinds of information and have different strengths and weaknesses with respect to the underlying model, we use corroboration of significant results across methods as our criteria for prioritization (at least two methods significant at $\mathrm{p}<0.05)$. Most of the corroborative results found were from the likelihood-based intrafamilial test of association (ASSOC) and tiled regression, which identifies the set of independent variants in the context of all variants in a tile that best predict the degree of lateral curvature.

Given the large number of SNPs tested, the multiple phenotypes used, and the number of association tests carried out, none of the SNPs were significant after adjusting for multiple tests (Bonferroni significance level of $p=0.00002$ ). Association analyses identified several SNPs significant at the nominal $\mathrm{p}<0.01$ level which were within the linkage peaks, some approaching but not reaching Bonferroni significance. Four SNPs on chromosome 9 and 4 SNPs on chromosome 16 met our prioriti- zation criteria (tables 2, 4). Six of the SNPs are located in genes or in characterized regions, resulting in 5 candidate genes including FAM129B and CERCAM on chromosome 9 and SYT17, GNAO1, and CDH3 on chromosome 16 .

On chromosome 9, rs2249110 lies in an intron of the FAM129B gene, a target of the MAP kinase-signaling cascade in human melanoma cells, and may play a role in apoptosis suppression [38]. rs7259 lies within the CERCAM gene, which produces a cerebral endothelial cell adhesion molecule potentially involved in leukocyte transmigration across the blood-brain barrier [39]. The last SNP, rs1871692, is not located in a gene, but is in a DNase-sensitive region indicative of a regulatory region [39]. This SNP is less than $350 \mathrm{~kb}$ from 2 SNPs (rs4979321 and rs891725) found to be mildly associated ( $p=0.0009$ and $\mathrm{p}=0.0003$, respectively) in a genome-wide association test of 419 families with IS [37].

On chromosome 16, rs229018 lies within the intron of SYT17, a member of the synaptotagmin protein family. These proteins are characterized by a specific $\mathrm{N}$-terminal transmembrane region, a variable linker, and two C-terminal domains and act in membrane trafficking between cells [39]. rs3785133 lies within the cadherin 3 (CDH3) gene, a member of the cadherin superfamily. The encoded protein is a calcium-dependent cell-cell adhesion glycoprotein comprised of five extracellular cadherin repeats, a transmembrane region, and a conserved cytoplasmic tail [40]. Aberrant expression of this protein is noted in some tissue-specific cancers. Mutations in the $\mathrm{CDH} 3$ gene are responsible for hypotrichosis with juvenile macular dystrophy, an autosomal recessive disorder characterized by sparse scalp hair and early blindness [41]. rs899234 is located within the intron of guanine nucleotide-binding protein, alpha (GNAO1). GNAO1, a heterotrimeric $G$ protein, is related to neuronal growth cone control and synaptic activities, but its specific functional roles are unknown [42]. This is notable given recent data suggestive of neural cell adhesion molecules and axonal guidance neurodevelopmental pathways as potential mechanisms contributing to IS pathogenesis [37].

Like many other complex diseases (e.g., breast cancer, diabetes, familial hypercholesterolemia) that initially had fairly substantial linkage peaks, no definitive associations were found. The failure of the intra-familial tests of association to identify a single definitive SNP that was responsible for most of the linkage signal was not unexpected. This would only be the case if FIS was genetically homogeneous and caused by a single simple Mendelian variant. Results from segregation analyses, linkage anal- 
yses, and genome-wide association studies for FIS suggest that is clearly not the case. Most likely, FIS is genetically heterogeneous, probably not simply caused by common variants but rather by both common and rare variants that differ from family to family.

In summary, we have utilized high-density SNP genotyping panels and statistical analyses to confirm previous work showing a significant relationship between loci on chromosomes 9 and 16 and the FIS phenotype and to prioritize candidate genes for either targeted, wholeexome, or whole-genome next generation sequencing. Ultimately, these findings will lead to the sequencing of regions under the linkage peaks and the identification of both common and rare variants within genes and regulatory regions which are related to the pathophysiology of FIS.

\section{Acknowledgements}

This work was supported by the Center for Inherited Disease Research (federal contract from the National Institutes of Health to Johns Hopkins University, contract No. N01-HG-65403); S.A.G.E. (National Center for Research Resources grant 1 P41 RR03655); LARRK Foundation (private donation); Institute de France Foundation Yves Cotrel; National Institutes of Health (R01AR048862-01A1), and the Division of Intramural Research of the National Human Genome Research Institute, National Institutes of Health. Additional SNP genotypes were performed at The SNP Center, Genetic Resources Core Facility, McKusick/Nathans Institute of Genetic Medicine, Johns Hopkins School of Medicine.

\section{References}

1 Weinstein SL: Adolescent idiopathic scoliosis: prevalence and natural history; in Weinstein SL (ed): The Pediatric Spine: Principles and Practice. New York, Raven Press, 1994, pp 463-478.

2 Daffner SD, Beimesch CF, Wang JC: Geographic and demographic variability of cost and surgical treatment of idiopathic scoliosis. Spine (Phila Pa 1976) 2010;35:1165-1169.

-3 Wise CA, Barnes R, Gillum J, Herring JA, Bowcock AM, et al: Localization of susceptibility to familial idiopathic scoliosis. Spine 2000;25:2372-2380.

-4 Salehi LB, Mangino M, De Serio S, De Cicco D, Capon F, et al: Assignment of a locus for autosomal dominant idiopathic scoliosis (IS) to human chromosome 17p11. Hum Genet 2002;111:401-404.

5 Chan V, Fong GC, Luk KD, Yip B, Lee MK, et al: A genetic locus for adolescent idiopathic scoliosis linked to chromosome 19p13.3. Am J Hum Genet 2002;71:401-406.

6 Justice CM, Miller NH, Marosy B, Zhang J, Wilson AF: Familial idiopathic scoliosis: evidence of an X-linked susceptibility locus. Spine 2003;28:589-594.

7 Miller NH, Justice CM, Marosy B, Doheny KF, Pugh E, et al: Identification of candidate regions for familial idiopathic scoliosis. Spine 2005;30:1181-1187.

8 Gao X, Gordon D, Zhang D, Browne R, Helms C, et al: CHD7 gene polymorphisms are associated with susceptibility to idiopathic scoliosis. Am J Hum Genet 2007;80: 957-965.

-9 Ocaka L, Zhao C, Reed JA, Ebenezer ND, Brice G, et al: Assignment of two loci for autosomal dominant adolescent idiopathic scoliosis to chromosomes $9 \mathrm{q} 31.2-\mathrm{q} 34.2$ and 17q25.3-qtel. J Med Genet 2008;45:87-92.
10 Raggio CL, Giampietro PF, Dobrin S, Zhao C, Dorshorst D, et al: A novel locus for adolescent idiopathic scoliosis on chromosome 12p. J Orthop Res 2009;27:1366-1372.

-11 Alden KJ, Marosy B, Nzegwu N, Justice CM, Wilson AF, et al: Idiopathic scoliosis: identification of candidate regions on chromosome 19p13. Spine 2006;31:1815-1819.

-12 Takahashi Y, Kou I, Takahashi A, Johnson TA, Kono K, et al: A genome-wide association study identifies common variants near LBX1 associated with adolescent idiopathic scoliosis. Nat Genet 2011;43:1237-1240.

13 Fan YH, Song YQ, Chan D, Takahashi Y, Ikegawa S, et al: SNP rs11190870 near LBX1 is associated with adolescent idiopathic scoliosis in southern Chinese. J Hum Genet 2012;57:244-246.

14 Miller NH, Schwab DL, Sponseller PD, Manolio TA, Pugh EW, et al: Characterization of idiopathic scoliosis in a clinically well-defined population. Clin Orthop Relat Res 2001;392:349-357.

15 Kane WJ: Scoliosis prevalence: a call for a statement of terms. Clin Orthop Relat Res 1977; 126:43-46.

16 Pruijs JE, Hageman MA, Keessen W, van der Meer R, van Wieringen JC: Variation in Cobb angle measurements in scoliosis. Skeletal Radiol 1994;23:517-520.

17 Mehta SS, Modi HN, Srinivasalu S, Chen T, Suh SW, et al: Interobserver and intraobserver reliability of Cobb angle measurement: endplate versus pedicle as bony landmarks for measurement: a statistical analysis. J Pediatr Orthop 2009;29:749-754.

18 Sambrook J, Fritsch EF, Maniatis T: Molecular Cloning: A Laboratory Manual. Cold Spring Harbor, Cold Spring Harbor Laboratory Press, 1989.
19 Fan JB, Oliphant A, Shen R, Kermani BG, Garcia F, et al: Highly parallel SNP genotyping. Cold Spring Harb Symp Quant Biol 2003;68:69-78

20 Gunderson KL, Kruglyak S, Graige MS, Garcia F, Kermani BG, et al: Decoding randomly ordered DNA arrays. Genome Res 2004; 14:870-877.

21 S.A.G.E. Statistical Analysis for Genetic Epidemiology. ed 6.0.1, 2009. http://darwin. cwru.edu/.

22 Boehnke M, Cox NJ: Accurate inference of relationships in sib-pair linkage studies. Am J Hum Genet 1997;61:423-429.

23 Broman KW, Weber JL: Estimation of pairwise relationships in the presence of genotyping errors. Am J Hum Genet 1998;63: 1563-1564.

24 O'Connell JR, Weeks DE: PedCheck: a program for identification of genotype incompatibilities in linkage analysis. Am J Hum Genet 1998;63:259-266

25 Wigginton JE, Cutler DJ, Abecasis GR: A note on exact tests of Hardy-Weinberg equilibrium. Am J Hum Genet 2005;76:887-893.

26 Barrett JC, Fry B, Maller J, Daly MJ: Haploview: analysis and visualization of $\mathrm{LD}$ and haplotype maps. Bioinformatics 2005;21: 263-265.

27 Pugh EW, Mandal DM, Wilson AF: A graphical approach for presenting linkage results from a genomic screen. Genet Epidemiol 1995;12:807-812.

28 Rabinowitz D, Laird N: A unified approach to adjusting association tests for population admixture with arbitrary pedigree structure and arbitrary missing marker information. Hum Hered 2000;50:211-223. 
29 Laird NM, Horvath S, Xu X: Implementing a unified approach to family-based tests of association. Genet Epidemiol 2000;19(suppl 1):S36-S42.

- 30 Horvath S, Xu X, Lake SL, Silverman EK, Weiss ST, et al: Family-based tests for associating haplotypes with general phenotype data: application to asthma genetics. Genet Epidemiol 2004;26:61-69.

31 Lake SL, Blacker D, Laird NM: Family-based tests of association in the presence of linkage. Am J Hum Genet 2000;67:1515-1525.

32 Wilson AF, Kim Y, Sung H, Cai JL, McMahon FJ, et al: Tiled regression: the use of regression methods in hotspot defined genomic segments to identify independent genetic variants responsible for variation in quantitative traits. Abstract 142. In: 'Abstracts from the Eighteenth Annual Meeting of the International Genetic Epidemiology Society', Genetic Epidemiology, 33:793, 2009.
33 Kim Y, Justice C, Sung H, Cai JL, Sorant AJM, et al: Tests of Association for Family Data: Tiled Regression with Generalized Estimation Equations. Abstract 163. In: 'Abstracts from the Nineteenth Annual Meeting of the International Genetic Epidemiology Society', Genetic Epidemiology, 34:984-985, 2010.

34 Sorant AJM, Cai JL, Sung H, Kim Y, Wilson AF: TiledReg: Software Implementation of Tiled Regression. Abstract 244. In: 'Abstracts from the Nineteenth Annual Meeting of the International Genetic Epidemiology Society', Genetic Epidemiology, 34:984-985, 2010.

35 Sung H, Kim Y, Cai J, Cropp CD, Simpson $\mathrm{CL}$, et al: Comparison of results from tests of association in unrelated individuals with uncollapsed and collapsed sequence variants using tiled regression. BMC Proc 2011;5 (suppl 9):S15.

36 Zeger SL, Liang KY: Longitudinal data analysis for discrete and continuous outcomes. Biometrics 1986;42:121-130.

37 Sharma S, Gao X, Londono D, Devroy SE, Mauldin KN, et al: Genome-wide association studies of adolescent idiopathic scoliosis suggest candidate susceptibility genes. Hum Mol Genet 2011;20:1456-1466.
8 Chen S, Evans HG, Evans DR: FAM129B/ MINERVA, a novel adherens junction-associated protein, suppresses apoptosis in HeLa cells. J Biol Chem 2011;286:10201-10209.

- 39 Rosenbloom KR, Dreszer TR, Pheasant M, Barber GP, Meyer LR, et al: ENCODE wholegenome data in the UCSC Genome Browser. Nucleic Acids Res 2010;38:D620-D625.

40 Shimoyama Y, Yoshida T, Terada M, Shimosato $\mathrm{Y}, \mathrm{Abe} \mathrm{O}$, et al: Molecular cloning of a human Ca2+-dependent cell-cell adhesion molecule homologous to mouse placental cadherin: its low expression in human placental tissues. J Cell Biol 1989;109:17871794.

41 Jelani M, Salman Chishti M, Ahmad W: A novel splice-site mutation in the $\mathrm{CDH} 3$ gene in hypotrichosis with juvenile macular dystrophy. Clin Exp Dermatol 2009;34:68-73.

-42 Nishimura-Akiyoshi S, Niimi K, Nakashiba T, Itohara S: Axonal netrin-Gs transneuronally determine lamina-specific subdendritic segments. Proc Natl Acad Sci USA 2007; 104:14801-14806. 